\title{
CyberKnife with tumor tracking: an effective treatment for high-risk surgical patients with stage I non-small cell lung cancer
}

\section{Viola J. Chen ${ }^{1}$, Eric Oermann ${ }^{1}$, Saloomeh Vahdat ${ }^{2}$, Jennifer Rabin ${ }^{1}$, Simeng Suy ${ }^{1}$, Xia Yu ${ }^{1}$, Sean P. Collins ${ }^{1}$, Deepa Subramaniam ${ }^{3}$, Filip Banovac ${ }^{4}$, Eric Anderson ${ }^{5}$ and Brian T. Collins ${ }^{1}{ }^{*}$}

1 Department of Radiation Medicine, Georgetown University Hospital, Washington, DC, USA

2 Department of Pathology, Georgetown University Hospital, Washington, DC, USA

${ }^{3}$ Division of Hematology and Oncology, Georgetown University Hospital, Washington, DC, USA

${ }^{4}$ Department of Radiology, Georgetown University Hospital, Washington, DC, USA

${ }^{5}$ Division of Pulmonary, Critical Care and Sleep Medicine, Georgetown University Hospital, Washington, DC, USA

\section{Edited by:}

Joe O'Sullivan, Queen's University

Belfast, UK

\section{Reviewed by:}

Joshua Silverman, New York University Medical Center, USA Jaroslaw T. Hepel, Rhode Island Hospital/Brown University, USA

*Correspondence:

Brian T. Collins, Department of Radiation Medicine, Georgetown University Hospital, Lower Level Bles Building, 3800 Reservoir Road Northwest, Washington, DC 20007, USA.

e-mail:collinsb@gunet.georgetown. edu
Published data suggests that wedge resection for stage I non-small cell lung cancer (NSCLC) is associated with improved overall survival compared to stereotactic body radiation therapy. We report CyberKnife outcomes for high-risk surgical patients with biopsyproven stage I NSCLC. PET/CT imaging was completed for staging. Three-to-five gold fiducial markers were implanted in or near tumors to serve as targeting references. Gross tumor volumes (GTVs) were contoured using lung windows; the margins were expanded by $5 \mathrm{~mm}$ to establish the planning treatment volume (PTV). Treatment plans were designed using a mean of 156 pencil beams. Doses delivered to the PTV ranged from 42 to $60 \mathrm{~Gy}$ in three fractions. The $30 \mathrm{~Gy}$ isodose contour extended at least $1 \mathrm{~cm}$ from the GTV to eradicate microscopic disease. Treatments were delivered using the CyberKnife system with tumor tracking. Examination and PET/CT imaging occurred at 3 month follow-up intervals. Forty patients (median age 76) with a median maximum tumor diameter of $2.6 \mathrm{~cm}$ (range, $1.4-5.0 \mathrm{~cm}$ ) and a mean post-bronchodilator percent predicted forced expiratory volume in $1 \mathrm{~s}$ (FEV1) of $57 \%$ (range, 21-111\%) were treated. A median dose of $48 \mathrm{~Gy}$ was delivered to the PTV over 3-13 days (median, 7 days). The $30 \mathrm{~Gy}$ isodose contour extended a mean $1.9 \mathrm{~cm}$ from the GTV. At a median 44 months (range, 12-72 months) follow-up, the 3 year Kaplan-Meier locoregional control and overall survival estimates compare favorably with contemporary wedge resection outcomes at 91 and 75\%, respectively. CyberKnife is an effective treatment approach for stage I NSCLC that is similar to wedge resection, eradicating tumors with $1-2 \mathrm{~cm}$ margins in order to preserve lung function. Prospective randomized trials comparing CyberKnife with wedge resection are necessary to confirm equivalence.

Keywords: non-small cell lung cancer, CyberKnife, stereotactic body radiation therapy, wedge resection

\section{INTRODUCTION}

Standard therapy for operable stage I non-small cell lung cancer (NSCLC) is lobectomy (Ginsberg and Rubinstein, 1995). This surgery requires the complete removal of the involved lobe plus ipsilateral hilar and mediastinal lymph node dissection. Consequently, locoregional recurrence is uncommon following lobectomy. Unfortunately, lobectomy is a major operation often associated with clinically meaningful declines in pulmonary function (Ginsberg and Rubinstein, 1995), multiple potentially debilitating morbidities (Handy et al., 2002), and infrequently death (Allen et al., 2006). Sublobar resection (segmentectomy or wedge resection) with adequate margins $(1-2 \mathrm{~cm})$ or adjuvant brachytherapy has been advocated for high-risk surgical patients with small peripheral lesions (Narsule et al., 2011). Such treatment in appropriately selected patients provides acceptable locoregional control without the early mortality and potentially clinically meaningful decline in lung function associated with lobectomy (Lee et al., 2003; Santos et al., 2003).

Historically, treatment outcomes for inoperable patients with clinical stage I NSCLC have been poor (Qiao et al., 2003). Both locoregional control and survival are typically disappointing with conventional radiation therapy techniques. For example, a study of conventionally fractionated radiation therapy studies found locoregional control rates ranging from 30 to $81 \%$ and a mean 5 year overall survival rate of $21 \%$ (Qiao et al., 2003). The development of stereotactic body radiation therapy (SBRT), capable of treating lung tumors with tight margins $(1 \mathrm{~cm})$, has improved outcomes (Lax et al., 2006). SBRT trials, utilizing adequate tumor doses, have consistently reported locoregional control rates comparable to surgery with locoregional control rates ranging from 78 to $87 \%$ and 3 year overall survival rates of $55-88 \%$ (Timmerman et al., 2003, 2006; Onishi et al., 2004; Mcgarry et al., 2005). 
Nonetheless, recently reported overall survival rates for high-risk surgical patients remain poor with such techniques (Grills et al., 2010; Timmerman et al., 2010). This poor survival has for the most part been attributed to comorbid illness rather than lung cancer progression; treatment related lung damage has not been implicated as a cause of mortality in these patients as initially predicted despite the generally high-lung doses delivered and the severe baseline pulmonary dysfunction reported.

We began treating stage I NSCLC patients in late 2004 using the CyberKnife ${ }^{\circledR}$ frameless robotic radiosurgery system (Accuray Incorporated, Sunnyvale, CA, USA) with Synchrony ${ }^{\circledR}$ tumor tracking using fiducials (Collins et al., 2007, 2009). The goal of such treatment was to maintain the impressive locoregional control rates of SBRT while further decreasing radiation induced lung damage and potentially improving survival. Continuous tracking of respiratory tumor motion and highly accurate beam alignment throughout treatment with the CyberKnife allowed us to deliver dose distributions with tighter margins on the gross tumor volume (GTV) than historically feasible (5 mm; Hoogeman et al., 2009). Numerous pencil beams were used to produce dose gradients that conform closely to the shape of the target, resulting in theoretically adequate microscopic disease doses extending $1 \mathrm{~cm}$ or more from the tumor (Papiez et al., 2003). The goal was similar to that of wedge resection, i.e., to reliably eradicate tumors with the minimum necessary margin to preserve lung function (Goldstein et al., 2003). We report intermediate outcomes for 40 consecutive highrisk surgical patients with clinical stage I NSCLC treated using this novel treatment approach at Georgetown University Hospital.

\section{MATERIALS AND METHODS \\ ELIGIBILITY}

The Medstar Health Research Institute - Georgetown University Oncology institutional review board approved this study and all participants provided informed written consent. The multidisciplinary thoracic oncology team evaluated patients. High-risk surgical patients with pathologically confirmed clinical Stage I NSCLC measuring $5 \mathrm{~cm}$ or less in maximum diameter were considered for protocol treatment. High-risk was defined as a postbronchodilator percent predicted forced expiratory volume in $1 \mathrm{~s}$ (FEV1) of less than 50\%, a carbon monoxide diffusing capacity (DLCO) of less than $50 \%$, age greater than 75 , or severe comorbid medical conditions. Prior to treatment, CT imaging of the chest and abdomen with IV contrast, PET imaging, and routine pulmonary function tests (PFTs) were completed when feasible. Mediastinoscopy was not performed for staging. Candidates were excluded from protocol treatment if fiducials could not be safely placed for tumor tracking or predetermined critical structure maximum radiation point dose limits could not be adhered to during treatment planning (Table 1).

\section{FIDUCIAL PLACEMENT}

With conscious sedation and local anesthesia, three to five gold fiducials measuring $0.8-1 \mathrm{~mm}$ in diameter by $3-7 \mathrm{~mm}$ in length (Item 351-1 Best Medical International Inc., Springfield, VA, USA) were placed with adequate spacing $(1-2 \mathrm{~cm})$ in or near tumors under CT-guidance as previously described (Banovac et al., 2007; Yousefi et al., 2007).
Table 1 | Critical structure radiation dose limits.

\begin{tabular}{ll}
\hline Adjacent structure & $\begin{array}{l}\text { Maximum dose limit } \\
\text { (total for three fractions; Gy) }\end{array}$ \\
\hline Spinal cord & 18 \\
Esophagus & 27 \\
Heart & 30 \\
Main bronchus & 30 \\
Trachea & 30 \\
Great vessels & 40 \\
\hline
\end{tabular}

\section{TREATMENT PLANNING}

Fine-cut $(1.25 \mathrm{~mm})$ treatment planning CTs were obtained 710 days after fiducial placement during a full inhalation breathhold. GTVs were contoured utilizing lung windows. The GTV margin was expanded $5 \mathrm{~mm}$ to establish the planning treatment volume (PTV). All critical central thoracic structures (Table 1) and the lungs were contoured to ensure that incidental radiation delivered to these structures was limited. A treatment plan was generated using the CyberKnife non-isocentric, inverse-planning ray-tracing algorithm with tissue density heterogeneity corrections for lung. Lower doses within the range of 42-60 Gy in three fractions were prescribed when concerns about adjacent critical structures arose and when patients were felt to have severe pulmonary dysfunction. The radiation was delivered to an isodose line that covered at least $95 \%$ of the PTV and resulted in the $30 \mathrm{~Gy}$ isodose contour extending a minimum of $1 \mathrm{~cm}$ from the GTV. As described in detail elsewhere (Collins et al., 2009), this approach is similar to sublobar resection which eliminates the tumor with $1 \mathrm{~cm}$ or greater margin to ensure that microscopic tumor extension is effectively treated. The percentage of the total lung volume receiving 15 Gy or more (V15) was limited to $15 \%$.

\section{TREATMENT DELIVERY}

Patients were treated according to the Georgetown University Hospital small peripheral pulmonary nodule protocol as previously described (Collins et al., 2007). Briefly, patients were brought to the CyberKnife suite and laid supine on the treatment table with their arms at their side. Three red light-emitting diodes (LEDs) were placed on the patient's anterior torso directed toward the camera array. Fiducials were located using orthogonal X-ray imagers. A correlation model was created between the LEDs tracked continuously by the camera array and fiducial positions imaged periodically by the X-ray targeting system. During treatment delivery the tumor position was tracked using the live camera array signal and correlation model; the linear accelerator was moved by the robotic arm to maintain precise alignment with the tumor throughout the respiratory cycle during radiation delivery. Fiducials were imaged prior to the delivery of every third beam to verify targeting accuracy and to update the correlation model.

\section{FOLLOW-UP STUDIES}

Examination and PET/CT imaging were performed at 3 month follow-up intervals. Locoregional recurrence was defined as progression in the involved lobe or regional lymph nodes per serial PET/CT imaging as previously reported (Vahdat et al., 2010). 
Other failures were considered distant. Biopsy was required to confirm progression.

\section{STATISTICAL ANALYSIS}

Data was analyzed and graphs were prepared with the SPSS 16.02 statistical package (IBM Corporation, Armonk, NY, USA). The follow-up duration was defined as the time from the date of completion of CyberKnife treatment to the last date of follow-up or the date of death. Actuarial locoregional control and overall survival were calculated using the Kaplan-Meier method.

\section{RESULTS}

\section{PATIENT AND TUMOR CHARACTERISTICS}

Forty consecutive patients ( 16 men and 24 women) with clinical stage I NSCLC (19 adenocarcinoma, 12 squamous cell carcinoma, and 9 not otherwise specified NSCLC) and an Eastern Cooperative Oncology Group (ECOG) performance status of two or less were treated over a 60 month period extending from November 2004 to 2009 (Table 2). No patients were lost to follow-up. Ninetyfive percent of the patients were smokers; pulmonary dysfunction was the primary rationale for non-surgical treatment. The mean post-bronchodilator percent predicted FEV1 was 57\% (range, 21$111 \%$ ). The mean maximum tumor diameter was $2.87 \mathrm{~cm}$ (range, $1.40-5.00 \mathrm{~cm})$.

\section{TREATMENT CHARACTERISTICS}

Treatment plans were composed of a mean of 156 pencil beams (Table 3). A median dose of $48 \mathrm{~Gy}$ was delivered to the prescription isodose line in three $1-2 \mathrm{~h}$ treatments over a 5-11 day period (mean, 7 days). The $30 \mathrm{~Gy}$ isodose contour, equivalent to $50 \mathrm{~Gy}$ in 25 fractions (Barton, 1995), extended a mean distance of $1.9 \mathrm{~cm}$ from the GTV to treat microscopic disease. The percent of the total lung volume receiving 15 Gy or more was 6.4\% (range, 2.2-13.6\%). No patient received adjuvant chemotherapy.

\section{DISEASE SPREAD AND SURVIVAL}

All patients with persistent tumor $\mathrm{SUV}_{\max }$ elevations following treatment were biopsied. Two local, one regional, and six distant failures were pathologically confirmed during follow-up. At a median follow-up of 44 months (range, 12-72 months), the 3 year Kaplan-Meier locoregional control and overall survival estimates were 91 and 75\%, respectively (Figures 1 and 2). Ten of 12 deaths were attributed to pulmonary dysfunction. Two patients developed progressive metastatic disease which resulted in their death during the third follow-up year.

\section{DISCUSSION}

Grills et al. (2010) published the results of an institutional retrospective analysis comparing SBRT with wedge resection for high-risk surgical patients with stage I NSCLC. This study was valuable because it was the first study to compare current SBRT and wedge resection outcomes. Increasingly, high-risk surgical patients are considering these options with minimal data available to aide decision-making. Although treatment arms were not randomized, known prognostic factors were similar but consistently inferior for the SBRT group. The primary finding of the Grills study was that locoregional control at 30 months was improved
Table 2 | Patient and tumor characteristics.

\begin{tabular}{|c|c|}
\hline FEV1, L & \\
\hline Mean & 1.22 \\
\hline Range & $0.53-3.06$ \\
\hline \multicolumn{2}{|l|}{ PREDICTED FEV1 (\%) } \\
\hline Mean & 57 \\
\hline Range & $21-111$ \\
\hline \multicolumn{2}{|l|}{ DLCO (mL/min/mmHg) } \\
\hline Mean & 9.99 \\
\hline Range & $3.49-23.3$ \\
\hline \multicolumn{2}{|c|}{ MEAN \% PREDICTED DLCO } \\
\hline Mean & 55 \\
\hline Range & $14-128$ \\
\hline \multicolumn{2}{|l|}{ ECOG } \\
\hline Median & 1 \\
\hline Range & $0-2$ \\
\hline \multicolumn{2}{|l|}{ AGE (YEARS) } \\
\hline Median & 76 \\
\hline Range & $63-87$ \\
\hline \multicolumn{2}{|l|}{ SEX (\%) } \\
\hline Male & 40 \\
\hline Female & 60 \\
\hline \multicolumn{2}{|l|}{ RACE (\%) } \\
\hline Caucasian & 82.5 \\
\hline African & 17.5 \\
\hline \multicolumn{2}{|l|}{ SMOKER (\%) } \\
\hline Yes & 95 \\
\hline No & 5 \\
\hline \multicolumn{2}{|l|}{ HISTOLOGY (\%) } \\
\hline Adenocarcinoma & 48 \\
\hline Squamous cell carcinoma & 30 \\
\hline NSCLC NOS & 22 \\
\hline \multicolumn{2}{|l|}{ CLINICAL STAGE (\%) } \\
\hline T1No & 60 \\
\hline T2NO & 40 \\
\hline \multicolumn{2}{|c|}{ MAXIMUM DIAMETER (cm) } \\
\hline Mean & 2.87 \\
\hline Median & 2.60 \\
\hline Range & $1.40-5.00$ \\
\hline
\end{tabular}

Table 3 | Treatment characteristics.

\begin{tabular}{ll}
\hline & Mean (range) \\
\hline Prescribed dose (Gy) in three treatments & $50(42-60)$ \\
Prescription isodose line (\%) & $80(74-85)$ \\
30 Gy isodose contour mean distance from the GTV (cm) & $1.90(1.08-2.74)$ \\
Number of beams per treatment & $156(79-242)$ \\
Number of paired X-ray verification images per treatment & $52(26-81)$ \\
Treatment course (days) & $7(5-11)$ \\
\% Total lung volume receiving 15 Gy or more & $6.4(2.2-13.6)$
\end{tabular}

with SBRT (91 vs. 73\%) and therefore SBRT was a reasonable treatment option for high-risk surgical patients not eligible for anatomic lobectomy. However, the validity of this conclusion has been questioned. Overall survival at 30 months was statistically 


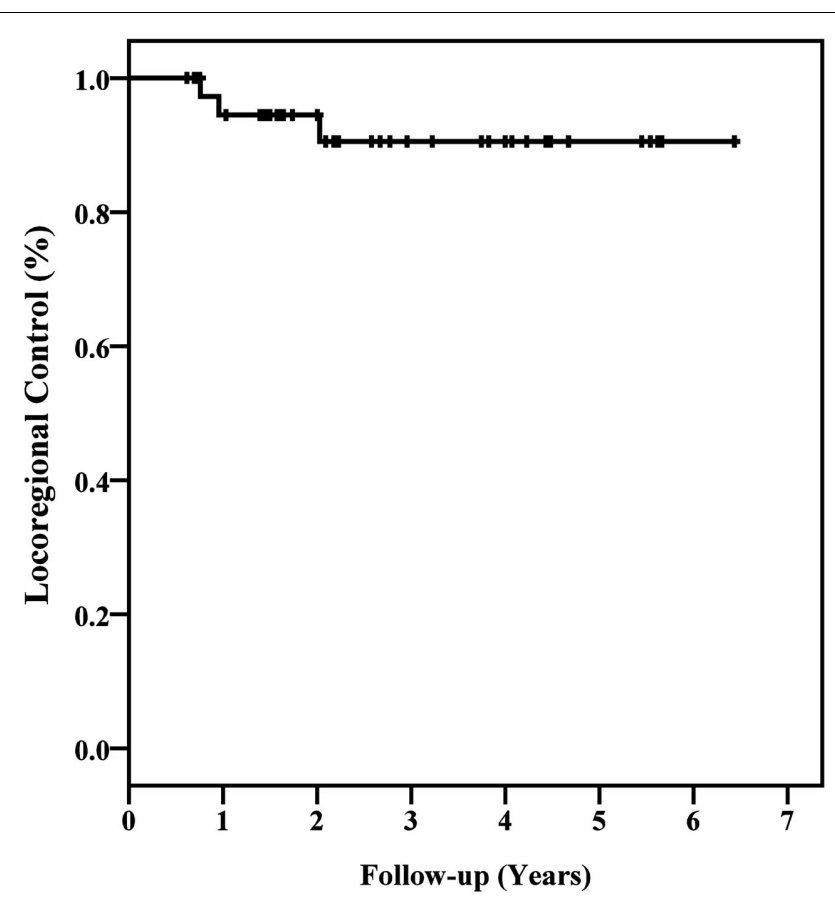

FIGURE 1 | Kaplan-Meier plot of locoregional control.

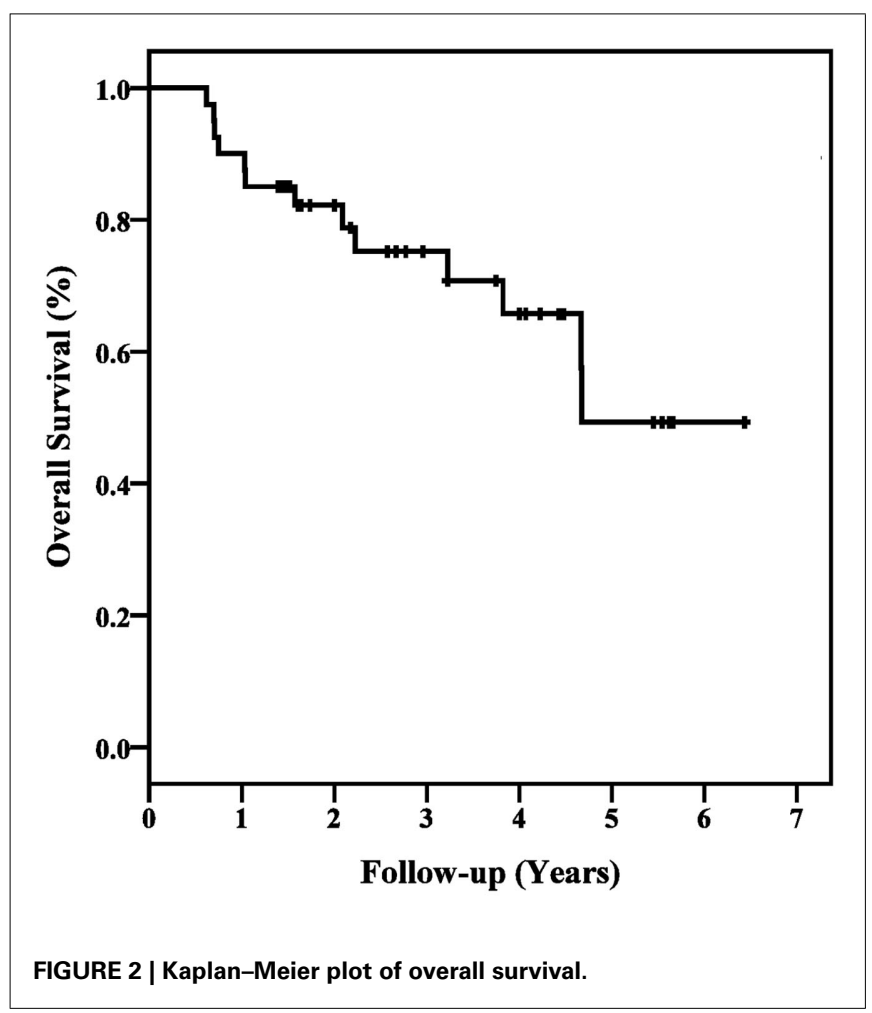

inferior in the SBRT group (72 vs. $87 \%$ ) conceivably enhancing SBRT's locoregional control.

In late 2004, we initiated a CyberKnife protocol for high-risk surgical patients with stage I NSCLC. Forty patients were treated in 60 months and followed for a minimum of 1 year. The goal was similar to that of wedge resection, i.e., to reliably eradicate tumors with the minimum necessary margin to preserve lung function. In the absence of comparative wedge resection results from our institution, we discuss the Grills et al. wedge resection outcomes here with respect to our outcomes. While a quantitative analysis has not been performed, the limited availability of data comparing SBRT to wedge resection and the desire for alternative treatment approaches for this high-risk surgical patient population compel us to explore the differences and similarities in these outcomes.

Baseline prognostic characteristics for the CyberKnife cohort were similar but generally inferior to the Grills et al. (2010) wedge resection cohort. However, the invasive fiducial placement requirement for CyberKnife treatment precluded the sickest patients from protocol therapy likely enhancing overall survival compared to the Grills et al. (2010) SBRT cohort. The 3 year locoregional control and overall survival estimates are comparable to the William Beaumont Hospital wedge resection cohort at 91 and $75 \%$, respectively (Grills et al., 2010). Based upon approximate values obtained from the survival curves reported by Grills et al., we conclude that CyberKnife treatment with tumor tracking results in locoregional control (91 vs. $\sim 70 \%$ ) and overall survival similar to wedge resection (75 vs. $\sim 80 \%$ ) for high-risk surgical patients with clinical stage I NSCLC. Both CyberKnife and wedge resection are reasonable treatment options for high-risk surgical patients not eligible for segmentectomy or lobectomy.

Critical issues concerning the validity of our observations do exist. It is widely held that PET/CT imaging surveillance promptly identifies locoregional recurrence following wedge resection. However, high peritumoral lung doses often result in focal radiation induced pneumonitis and fibrosis, hampering PET/CT recurrence assessment following irradiation (Vahdat et al., 2010). Routine biopsy was not completed in our cohort given the uncertain clinical significance of early transient elevations in tumor $\mathrm{SUV}_{\max }$ following radiation treatment and the risk associated with biopsy in this high-risk surgical patient population with limited salvage treatment options. Therefore, confirmation of radiographic impressions was limited to biopsy in three patients following a persistent increase in tumor $S U V_{\max }$; biopsies were not taken to confirm the absence of disease in cases in which tumor $\mathrm{SUV}_{\max }$ remained low or returned to the normal range following transient elevations. Therefore, it is likely that the CyberKnife 3 year locoregional control rate is less than our reported $91 \%$ rate.

Finally, the Grills et al. wedge patients discussed here are clearly biased against wedge resection. This group was retrospectively collected from the general patient population of the William Beaumont Hospital while the CyberKnife patients were selected for protocol therapy from the high-risk surgical patient population treated at Georgetown University Hospital. Unfortunately, to our knowledge, no contemporary single institution prospective wedge resection outcomes data exists. Ultimately, the results of randomized trials will dictate treatment recommendations. In the interim, we will continue to treat selected consenting high-risk surgical patients with clinical stage I NSCLC using CyberKnife if wedge resection is the only surgical treatment option. The present encouraging intermediate outcomes adequately support 
this practice in this frail patient population that characteristically dies of comorbid illnesses rather than tumor progression.

\section{CONCLUSION}

CyberKnife is an effective treatment for stage I NSCLC. The approach is similar to that of wedge resection, eradicating tumors

\section{REFERENCES}

Allen, M. S., Darling, G. E., Pechet, T. T., Mitchell, J. D., Herndon, J. E. II., Landreneau, R. J., Inculet, R. I., Jones, D. R., Meyers, B. F., Harpole, D. H., Putnam, J. B. Jr., and Rusch, V. W. (2006). Morbidity and mortality of major pulmonary resections in patients with early-stage lung cancer: initial results of the randomized, prospective ACOSOG Z0030 trial. Ann. Thorac. Surg. 81, 1013-1019; discussion 1019-1020.

Banovac, F., Mcrae, D., Dieterich, S., Wong, K., Dias, L., and Chang, T. (2007). "Percutaneous placement of fiducial markers for thoracic malignancies," in Robotic Radiosurgery: Treating Tumors that Move with Respiration, eds H. C. Urschel, J. J. Kresel, J. D. Luketich, L. Papiez, and R. D. Timmerman (Berlin: SpringerVerlag), 15-29.

Barton, M. (1995). Tables of equivalent dose in $2 \mathrm{~Gy}$ fractions: a simple application of the linear quadratic formula. Int. J. Radiat. Oncol. Biol. Phys. 31, 371-378.

Collins, B. T., Erickson, K., Reichner, C. A., Collins, S. P., Gagnon, G. J., Dieterich, S., Mcrae, D. A., Zhang, Y., Yousefi, S., Levy, E., Chang, T., JamisDow, C., Banovac, F., and Anderson, E. D. (2007). Radical stereotactic radiosurgery with real-time tumor motion tracking in the treatment of small peripheral lung tumors. Radiat. Oncol. 2, 39.

Collins, B. T., Vahdat, S., Erickson, K., Collins, S. P., Suy, S., Yu, X., Zhang, Y., Subramaniam, D., Reichner, C. A., Sarikaya, I., Esposito, G., Yousefi, S., Jamis-Dow, C., Banovac, F., and Anderson, E. D. (2009). Radical CyberKnife radiosurgery with tumor tracking: an effective treatment for inoperable small peripheral stage I non-small cell lung cancer. J. Hematol. Oncol. 2, 1.

Ginsberg, R. J., and Rubinstein, L. V. (1995). Randomized trial of lobectomy versus limited resection for $\mathrm{T} 1$ NO non-small cell lung cancer. Lung Cancer Study Group. Ann. Thorac. Surg. 60, 615-622; discussion 622-613.
Goldstein, N. S., Ferkowicz, M., Kestin, L., Chmielewski, G. W., and Welsh, R. J. (2003). Wedge resection margin distances and residual adenocarcinoma in lobectomy specimens. Am. J. Clin. Pathol. 120, 720-724.

Grills, I. S., Mangona, V. S., Welsh, R., Chmielewski, G., Mcinerney, E., Martin, S., Wloch, J., Ye, H., and Kestin, L. L. (2010). Outcomes after stereotactic lung radiotherapy or wedge resection for stage I nonsmall-cell lung cancer. J. Clin. Oncol. 28, 928-935.

Handy, J. R. Jr., Asaph, J. W., Skokan, L., Reed, C. E., Koh, S., Brooks, G., Douville, E. C., Tsen, A. C., Ott, G. Y., and Silvestri, G. A. (2002). What happens to patients undergoing lung cancer surgery? Outcomes and quality of life before and after surgery. Chest 122, 21-30.

Hoogeman, M., Prevost, J. B., Nuyttens, J., Poll, J., Levendag, P., and Heijmen, B. (2009). Clinical accuracy of the respiratory tumor tracking system of the CyberKnife: assessment by analysis of $\log$ files. Int. J. Radiat. Oncol. Biol. Phys. 74, 297-303.

Lax, I., Panettieri, V., Wennberg, B., Amor Duch, M., Naslund, I., Baumann, P., and Gagliardi, G. (2006). Dose distributions in SBRT of lung tumors: comparison between two different treatment planning algorithms and Monte-Carlo simulation including breathing motions. Acta Oncol. 45, 978-988.

Lee, W., Daly, B. D., Dipetrillo, T. A., Morelli, D. M., Neuschatz, A. C., Morr, J., and Rivard, M. J. (2003). Limited resection for nonsmall cell lung cancer: observed local control with implantation of I125 brachytherapy seeds. Ann. Thorac. Surg. 75, 237-242; discussion 242-233.

Mcgarry, R. C., Papiez, L., Williams, M., Whitford, T., and Timmerman, R. D. (2005). Stereotactic body radiation therapy of early-stage nonsmall-cell lung carcinoma: phase I study. Int. J. Radiat. Oncol. Biol. Phys. 63, 1010-1015.

Narsule, C. K., Ebright, M. I., and Fernando, H. C. (2011). Sublobar versus

with 1-2 cm margins in order to preserve lung function. Locoregional control and overall survival outcomes appear similar to contemporary wedge resection results. Prospective randomized trials comparing CyberKnife with wedge resection for high-risk surgical patients with stage I NSCLC are necessary to confirm equivalence.

lobar resection: current status. Cancer J. 17, 23-27.

Onishi, H., Araki, T., Shirato, H., Nagata, Y., Hiraoka, M., Gomi, K., Yamashita, T., Niibe, Y., Karasawa, K., Hayakawa, K., Takai, Y., Kimura, T., Hirokawa, Y., Takeda, A., Ouchi, A., Hareyama, M., Kokubo, M., Hara, R., Itami, J., and Yamada, K. (2004). Stereotactic hypofractionated high-dose irradiation for stage I nonsmall cell lung carcinoma: clinical outcomes in 245 subjects in a Japanese multiinstitutional study. Cancer 101, 1623-1631.

Papiez, L., Timmerman, R., Desrosiers, C., and Randall, M. (2003). Extracranial stereotactic radioablation: physical principles. Acta Oncol. 42, 882-894.

Qiao, X., Tullgren, O., Lax, I., Sirzen, F., and Lewensohn, R. (2003). The role of radiotherapy in treatment of stage I non-small cell lung cancer. Lung Cancer 41, 1-11.

Santos, R., Colonias, A., Parda, D., Trombetta, M., Maley, R. H. Macherey, R., Bartley, S., Santucci, T., Keenan, R. J., and Landreneau, R. J. (2003). Comparison between sublobar resection and 125Iodine brachytherapy after sublobar resection in high-risk patients with stage I non-small-cell lung cancer. Surgery 134, 691-697; discussion 697.

Timmerman, R., Mcgarry, R., Yiannoutsos, C., Papiez, L., Tudor, K., Deluca, J., Ewing, M., Abdulrahman, R., Desrosiers, C., Williams, M., and Fletcher, J. (2006). Excessive toxicity when treating central tumors in a phase II study of stereotactic body radiation therapy for medically inoperable early-stage lung cancer. $J$. Clin. Oncol. 24, 4833-4839.

Timmerman, R., Papiez, L., Mcgarry, R. Likes, L., Desrosiers, C., Frost, S., and Williams, M. (2003). Extracranial stereotactic radioablation: results of a phase I study in medically inoperable stage I non-small cell lung cancer. Chest 124, 1946-1955.

Timmerman, R., Paulus, R., Galvin, J. Michalski, J., Straube, W., Bradley, J., Fakiris, A., Bezjak, A., Videtic, G., Johnstone, D., Fowler, J., Gore, E. and Choy, H. (2010). Stereotactic body radiation therapy for inoperable early stage lung cancer. JAMA 303, 1070-1076.

Vahdat, S., Oermann, E. K., Collins, S. P., Yu, X., Abedalthagafi, M. Debrito, P., Suy, S., Yousefi, S., Gutierrez, C. J., Chang, T., Banovac, F., Anderson, E. D., Esposito, G., and Collins, B. T. (2010). CyberKnife radiosurgery for inoperable stage IA non-small cell lung cancer: $18 \mathrm{~F}$ fluorodeoxyglucose positron emission tomography/computed tomography serial tumor response assessment. J. Hematol. Oncol. 3, 6.

Yousefi, S., Collins, B. T., Reichner, C. A., Anderson, E. D., Jamis-Dow, C., Gagnon, G., Malik, S., Marshall, B., Chang, T., and Banovac, F. (2007). Complications of thoracic computed tomography-guided fiducial placement for the purpose of stereotactic body radiation therapy. Clin. Lung Cancer 8, 252-256.

Conflict of Interest Statement: The authors declare that the research was conducted in the absence of any commercial or financial relationships that could be construed as a potential conflict of interest.

Received: 31 October 2011; accepted: 16 January 2012; published online: 01 February 2012.

Citation: Chen VJ, Oermann E, Vahdat S, Rabin J, Suy S, Yu X, Collins SP, Subramaniam D, Banovac F, Anderson $E$ and Collins BT (2012) CyberKnife with tumor tracking: an effective treatment for highrisk surgical patients with stage I nonsmall cell lung cancer. Front. Oncol. 2:9. doi: 10.3389/fonc.2012.00009

This article was submitted to Frontiers in Radiation Oncology, a specialty of Frontiers in Oncology.

Copyright (c) 2012 Chen, Oermann, Vahdat, Rabin, Suy, Yu, Collins, Subramaniam, Banovac, Anderson and Collins. This is an open-access article distributed under the terms of the Creative Commons Attribution Non Commercial License, which permits non-commercial use, distribution, and reproduction in other forums, provided the original authors and source are credited. 